\title{
Atuação dos Agentes Comunitários de Saúde para o controle da hanseníase: uma revisão de escopo
}

Community Health Workers performance for leprosy control: a scoping review

Actuación de los Agentes Comunitarios de Salud para el control de la lepra: una revisión de alcance

Natacha Bolorino

ORCID: https://orcid.org/0000-0002-3039-2987

Universidade Estadual de Londrina, Brasil

E-mail: natacha.bolorino@uel.br

Carla Fernanda Tiroli

ORCID: https://orcid.org/0000-0002-0974-9689

Universidade Estadual de Londrina, Brasil

E-mail: carla.fernanda@uel.br

Izabela Nayara Ricardo

ORDID: https://orcid.org/0000-0001-5290-9302 Universidade Estadual de Londrina, Brasil E-mail: izan.enf@gmail.com

Franciely Midori Bueno de Freitas

ORCID: https://orcid.org/0000-0003-0815-2765

Universidade Estadual de Londrina, Brasil

E-mail: franmidori@hotmail.com

Laís Cristina Gonçalves Ribeiro

ORCID: https://orcid.org/0000-0002-4522-3297

Universidade Estadual de Londrina, Brasil E-mail: lcg.enf@hotmail.com

Simone Cristina Castanho Sabaini de Melo

ORCID: https://orcid.org/0000-0002-4822-3591

Universidade Estadual do Norte do Paraná, Brasil

E-mail: simonecastanho@uenp.edu.br

Natalia Marciano de Araújo Ferreira

ORCID: https://orcid.org/0000-0002-5802-6188

Universidade Estadual de Londrina, Brasil E-mail: natty_fdj@hotmail.com

Vanessa Cristina Luquini

ORCID: https://orcid.org/0000-0002-6420-4446 Prefeitura Municipal de Ibiporã, Brasil E-mail: vanessaluquini.vcl@gmail.com

Rosângela Aparecida Pimenta Ferrari ORCID: https://orcid.org/0000-0003-0157-7461

Universidade Estadual de Londrina, Brasil E-mail: ropimentaferrari@uel.br

Rafaela Marioto Montanha

ORCID: https://orcid.org/0000-0002-7237-0110

Universidade Estadual de Londrina, Brasil

E-mail: rafaela.montanha@hotmail.com

Amanda Caroliny Gomilde

ORCID: https://orcid.org/0000-0002-9255-4128

Universidade Estadual de Londrina, Brasil

E-mail: amandagomilde1@gmail.com

João Victor Rodrigues Cardoso

ORCID: https://orcid.org/0000-0002-6505-7302

E-mail: joaoo.vrc@gmail.com

Universidade Estadual de Londrina, Brasil

Jamile Mariana da Fonseca Pereira

ORCID: https://orcid.org/0000-0001-6852-364X

Centro Universitário Filadélfia, Brasil

E-mail: jamile.mary@outlook.com

Flávia Meneguetti Pieri

ORCID: https://orcid.org/0000-0003-1239-2550

Universidade Estadual de Londrina, Brasil E-mail: fpieri@uel.br 


\title{
Resumo
}

Objetivo: Mapear e sintetizar as evidências científicas da atuação do agente comunitário de saúde para as ações de controle da hanseníase com base nos atributos da atenção primária à saúde. Metodologia: Revisão de escopo, conforme Joanna Briggs Institute e o PRISMA-ScR. A pesquisa foi realizada em bases de dados eletrônicas, na Biblioteca Virtual de Saúde, Cumulative Index to Nursing and Allied Health Literature (CINAHL), U.S National Library of Medicine National Institutes of Health (PubMed), Grey Literature Report e Google Acadêmico. A coleta de dados ocorreu em 07 de abril de 2021, com o limite temporal estabelecido entre 1991 a abril de 2021. Resultados: Foram identificadas 359 evidências científicas e após a aplicação as quatro etapas de inclusão, resultaram em 13 evidências. A maioria das pesquisas foram realizadas na região sudeste do Brasil entre os anos de 2005 a 2019 . As principais evidências encontradas foram categorizadas de acordo com os atributos. Porta de entrada: Cadastramento de casos, detecção de sinais e sintomas na comunidade e visita domiciliar. Atendimento continuado: Adesão ao tratamento, verificação de reações indesejadas e supervisão da dose diária. Integralidade: vigilância dos contatos intradomiciliares. Orientação familiar: orientações sobre o tratamento contínuo e visitas domiciliares para a realização de orientações. Orientação comunitária: reuniões para grupos, sensibilizações nas escolas e produção de material didático. Orientação profissional: treinamentos. Considerações Finais: Os resultados enfatizaram a necessidade de capacitação profissional e por outro lado, foi destacada a importância da sua atuação junto ao indivíduo, família, grupos e sociedade.

Palavras-chave: Revisão de escopo; Atenção Primária à Saúde; Agente comunitário de saúde; Conhecimentos, atitudes e prática em saúde; Hanseníase.

\begin{abstract}
Objective: To map and synthesize the scientific evidence of the performance of the community health worker based on the attributes of primary health care for leprosy control actions. Methodology: Scoping review, according to Joanna Briggs Institute and PRISMA-ScR. The research was carried out in electronic databases, in the Virtual Health Library, in the Cumulative Index to Nursing and Allied Health Literature (CINAHL), in the U.S National Library of Medicine, in the National Institutes of Health (PubMed), in the Gray Literature Report, and in the Google Scholar. Data collection took place on April 7, 2021, with the time limit established between 1991 and April 2021. Results: 359 scientific evidences were identified and after the application of the 4 stages of inclusion, resulted in 13 evidences. Most surveys were carried out in the southeastern region of Brazil between the years 2005 to 2019. The main evidence found was categorized according to the attribute. Gateway: Registration of cases, detection of signs and symptoms in the community, home visit to detect new cases, application of a questionnaire with questions about signs and symptoms. Continued care: Adherence to treatment, checking for unwanted reactions and supervision of the daily dose. Comprehensiveness: surveillance of household contacts. Family counseling: guidance on continuous treatment and home visits for guidance. Community orientation: group meetings, school sensitization and production of teaching material by the teaching and service team. Professional guidance: training. Final Considerations: The results emphasized the need for professional training and, on the other hand, the importance of their work with the individual, family, groups and society was highlighted.
\end{abstract}

Keywords: Scoping review; Primary Health Care; Community health worker; Health knowledge, attitudes and practice; Leprosy.

\section{Resumen}

Objetivo: Mapear y sintetizar las evidencias científicas de la actuación de los agentes comunitarios de salud para acciones de control de la lepra a partir de los atributos de la atención primaria de salud. Metodología: Revisión de alcance, según Joanna Briggs Institute y PRISMA-ScR. La investigación se realizó en bases de datos electrónicas, en la Virtual Health Library, en Cumulative Index to Nursing and Allied Health Literature (CINAHL), en la U.S National Library of Medicine, en National Institutes of Health (PubMed), en Grey Literature Report y en Google Académico. La recolección de datos ocurrió el 7 de abril de 2021, con el límite temporal establecido entre 1991 y abril de 2021. Resultados: Se identificaron 359 evidencias científicas y, después de aplicar las cuatro etapas de inclusión, se obtuvieron 13 evidencias. La mayoría de las investigaciones se realizó en la región sureste de Brasil entre los años 2005 y 2019. Las principales evidencias encontradas fueron categorizadas según los atributos. Puerta de entrada: registro de casos, detección de signos y síntomas en la comunidad y visita domiciliaria. Atención continuada: adhesión al tratamiento, verificación de reacciones no deseadas y supervisión de la dosis diaria. Integralidad: vigilancia de los contactos dentro de la residencia. Orientación familiar: orientaciones sobre el tratamiento continuo y visitas domiciliarias para realizar las orientaciones. Orientación comunitaria: encuentros de grupos, sensibilización en escuelas y producción de material didáctico. Orientación profesional: formaciones. Consideraciones finales: Los resultados enfatizaron la necesidad de capacitación profesional y, además, resaltó la importancia de su actuación junto al individuo, a la familia, a los grupos y a la sociedad.

Palabras clave: Revisión de alcance; Atención Primaria de Salud; Agente comunitario de salud; Conocimientos, actitudes y práctica en salud; Lepra. 


\section{Introdução}

Em 2019, a Organização Mundial da Saúde (OMS), caracteriza o cenário epidemiológico da hanseníase, obtendo o total de 202.185 casos novos da doença no mundo. Desses, 29.936 (93\%) ocorreram na região das Américas e 27.864 o Brasil foi responsável, sendo 1.545 (5,5\%) desses casos em menores de 15 anos, e entre os 23.843 (85,6\%) avaliados no momento do diagnóstico, 2.361 (9,9\%) apresentaram Grau de Incapacidade Física (GIF) 2, considerado um indicador relevante para avaliar o diagnóstico tardio (Brasil, 2021).

Ainda nesse cenário epidemiológico, destaca-se, o crescente percentual de casos novos Multibacilares (MB), passando de 59,1\% em 2010 para 78,4\% em 2019, o que corresponde a forma infectante da doença. De acordo com esse panorama, o Brasil ocupa o segundo lugar na relação de países com maior número de casos no mundo, atrás apenas da Índia (Brasil, 2021).

Entre as principais estratégias para alcançar a redução dessas taxas, baseia-se na identificação das necessidades em saúde das pessoas dentro de um contexto social e o conhecimento dessa realidade pela Estratégia Saúde da Família (ESF), sendo fundamental para o planejamento de ações estratégicas, racionalizando a organização dos serviços de Atenção Primária à Saúde (APS).

Nessa perspectiva, a Estratégia Nacional para o Enfrentamento da Hanseníase (ENEH) 2019-2022 traz a visão de um Brasil sem hanseníase, com o objetivo pautado em três pilares: 1) fortalecer a gestão do programa, 2) enfrentamento da doença e suas complicações e o terceiro, combater a discriminação e promover a inclusão. Ao reorganizarem o serviço a ESF para atender a esses pilares, devem levar em conta a organização de uma rede de saúde com a integração das ações de enfrentamento na APS e a manutenção da atenção especializada nos níveis secundário e terciário em razão do potencial incapacitante da doença (Araújo et al., 2017; Brasil, 2019).

De acordo com Starfield (2002), o papel da APS quando organizada em atributos essenciais (porta de entrada, acesso, atendimento continuado, integralidade dos serviços disponíveis e prestados, coordenação) e derivados (orientação familiar, orientação comunitária e competência cultural) poderá garantir qualidade no atendimento, prevenção, diagnóstico e tratamento precoce e redução de cuidados especializados.

Para a operacionalização das ações dos atributos, a APS tem como eixo estruturante a ESF, que conta com a composição de profissionais de conhecimentos multidisciplinares que objetivam atender às necessidades de saúde da população (Portaria nº 2436, 2017).

O protagonista da ESF é o Agente Comunitário de Saúde (ACS), que tem como seu principal cenário de atuação o ambiente familiar por território ou área de abrangência (Peruzzo et al., 2018). Tem subsídio para identificar e levantar os problemas da população que está sob sua responsabilidade com intermédio das fichas de cadastro familiar e individual da Estratégia e-SUS da Atenção Básica (AB) em nível nacional (Brasil, 2018).

Desse modo, justifica-se a realização dessa revisão de escopo que tem como objetivo: mapear e sintetizar as evidências científicas da atuação dos agentes comunitários de saúde para as ações de controle de hanseníase com base nos atributos da atenção primária à saúde.

\section{Metodologia}

A metodologia escolhida para esse estudo foi a revisão de escopo desenvolvida com base nas orientações metodológicas propostas por Arksey e O’Malley (2005) e refinada pelo Instituto Joanna Briggs [IJB] (Briggs, 2015), com informações organizadas conforme itens do Checklist para relatórios preferenciais de Revisões Sistemáticas (RS), extensões de Meta-análises e Revisões de Escopo (PRISMA-ScR) (Tricco et al., 2018). O protocolo dessa revisão foi publicado no periódico Research, Society and Development v. 9, n.9, e896998092 em 12 de setembro de 2020.

O desenvolvimento dessa revisão incluiu etapas definidas por Arksey e O’Malley (2005) refinadas por Levac et al. 
(2010), sendo cinco delas consideradas essenciais: (1) Identificação da pergunta de pesquisa; (2) Identificação de estudos relevantes; (3) Seleção de estudos para a revisão; (4) Mapeamento dos dados e (5) Coleta, resumo e síntese dos resultados.

Na primeira etapa foi estruturado a pergunta de pesquisa baseada na estratégia PCC, acrônimo para P (população): Agentes Comunitários de Saúde; C (Conceito): Atuação para as ações de controle e C (Contexto): hanseníase.

Com base nessa estratégia, elaborou-se as seguintes perguntas de pesquisa: (1) Quais as evidências científicas sobre a atuação do Agente Comunitário de Saúde para as ações de controle da hanseníase? (2) Especificamente essa atuação se enquadra em qual atributo da APS (porta de entrada, acesso, atendimento continuado, integralidade dos serviços disponíveis e prestados, orientação familiar, orientação comunitária e profissional)?

$\mathrm{Na}$ segunda etapa, os documentos foram identificados a partir de uma busca sistemática que foi concluída em 07 de abril de 2021 pela pesquisadora principal, utilizando o cruzamento de Descritores em Ciências da Saúde (DeCS), descritores não controlados e o Medical Subject Headings (MeSH), acessadas por meio da Comunidade Acadêmica Federada (CAFe), via Biblioteca Central da Universidade Estadual de Londrina (UEL).

Para estes descritores utilizaram-se o operador booleano "AND" resultando nas seguintes estratégias de busca: Biblioteca Virtual de Saúde (BVS): "Agente Comunitário de Saúde" AND "Conhecimentos, Atitudes e Práticas e Atitudes AND Hanseníase / "Agente Comunitário de Saúde" AND "Acesso aos serviços de saúde" AND Hanseníase/ "Agente Comunitário de Saúde" AND "Integralidade em Saúde" AND Hanseníase/ "Agente Comunitário de Saúde" AND "Prevenção \& Controle" AND Hanseníase / "Agente Comunitário de Saúde" AND "Prevenção Primária" AND Hanseníase / "Agente Comunitário de Saúde" AND Diagnose AND Hanseníase /“Agente Comunitário de Saúde" AND "Porta de entrada" AND Hanseníase / "Agente Comunitário de Saúde" AND "Atendimento continuado" AND Hanseníase / "Agente Comunitário de Saúde" AND "Orientação familiar" AND Hanseníase/ "Agente Comunitário de Saúde" AND "Orientação comunitária" AND Hanseníase / "Agente Comunitário de Saúde" AND "ações preventivas, promocionais e curativas AND Hanseníase / "Agente Comunitário de Saúde" AND "busca de comunicantes" AND Hanseníase/ "Agente Comunitário de Saúde" AND "busca de contatos" AND Hanseníase / "Agente Comunitário de Saúde" AND "grupo de autocuidado" AND Hanseníase / "Agente Comunitário de Saúde" AND Acompanhamento AND Hanseníase / "Agente Comunitário de Saúde" AND "Identificação de anormalidades" AND Hanseníase / "Agente Comunitário de Saúde" AND "Busca ativa" AND Hanseníase / "Agente Comunitário de Saúde" AND "identificação de sinais e sintomas" AND Hanseníase / "Agente Comunitário de Saúde" AND "Levantamento de suspeita” AND Hanseníase/“Agente Comunitário de Saúde" AND "Divulgação da hanseníase" AND Hanseníase / "Agente de Salud Comunitaria" AND "Conocimientos, Atitudes y Practica y Salud" AND Lepra / "Agente de Salud Comunitaria" AND "Acessibilidad a los Servicios de Salud" AND Lepra / "Agente de Salud Comunitaria" AND "Integralidad en Salud" AND Lepra / "Agente de Salud Comunitaria" AND "Prevención \& Control" AND Lepra / "Agente de Salud Comunitaria" AND "Prevención Primaria" AND Lepra / "Agente de Salud Comunitaria" AND Diagnóstico AND Lepra. Cumulative Index to Nursing and Allied Health Literature (CINAHL): "Comunity Health Worker" AND "Prevention and control" AND Leprosy / "Comunity Health Worker" AND "Primary Prevention" AND Leprosy / "Comunity Health Worker" AND "Diagnosis" AND Leprosy/ "Comunity Health Worker” AND "Leprosy prevention and control” AND Leprosy. U.S National Library of Medicine National Institutes of Health (PubMed): “Community Health Worker" AND "Health Knowledge, Actitudes, Pratice" AND "Leprosy" / "Community Health Worker" AND Acessibility AND Leprosy / "Community Health Worker" AND Integrality AND Leprosy / "Community Health Worker" AND "Prevention and control" AND Leprosy / "Community Health Worker" AND "Primary Prevention" AND Leprosy / "Community Health Worker" AND "Diagnosis" AND Mycobacterium. Grey Literature Report: "Community Health Worker” AND "Health Knowledge, Actitudes, Pratice" and "Leprosy". Google Acadêmico: "Agente comunitário de saúde" and "busca ativa" and Hanseníase / "Agente comunitário de saúde" and "Busca de contatos" and Hanseníase / "Agente comunitário de saúde" and "Busca de 
contatos" and Hanseníase / "Agente comunitário de saúde" and "prevenção primária" and Hanseníase.

As evidências recuperadas das bases de dados foram exportadas para o gerenciador de referências EndNote Online® para organização e padronização do formato de exportação aceitável no Software de gerenciador de revisões sistemáticas denominado State of the Art through Systematic Review (StArt ${ }^{\circledR}$ ), uma ferramenta utilizada em revisões sistemáticas, desenvolvida pelo Laboratório de Pesquisa de Engenharia de Software do Departamento de Computação da Universidade Federal de São Carlos (UFSCAR) (Fabbri et al., 2016).

$\mathrm{Na}$ terceira etapa, estabeleceu-se critérios com base na pergunta e pesquisa: (1) Estudos primários e secundários; (2) Idiomas português, inglês e espanhol; (3) Arquivos publicados a partir de 1991 até 07 de abril de 2021; (4) Teses e dissertações; (5) Resenhas, relatórios, revisões de livros, erratas e comentários de artigos; (6) Estudos brasileiros. E como critério de exclusão: (7) Arquivos completos não encontrados por meio de conta institucional; (8) Editais de seleção; (9) Não atender à pergunta de pesquisa; (10) Arquivos duplicados; (11) Fichas catalográficas e (12) Manuais de recomendações.

O recorte temporal foi determinado pelo fato desse ano (1991) ser considerado um marco histórico por ser instituído o Programa Agentes Comunitários de Saúde (PACS) no Brasil.

Outro ponto relevante para a seleção dos documentos diz respeito ao ACS como membro da equipe ESF, sendo assim, os documentos que tratassem de Ações de Controle da Hanseníase (ACH) pela equipe ESF, incluindo o ACS e enfatizando o seu papel na atuação foram incluídos.

No que tange à qualidade dos artigos e documentos científicos recuperados, a revisão de escopo não considera a análise quantitativa do rigor metodológico, sendo assim, esse critério não foi analisado (Briggs, 2015).

Ainda com relação à seleção dos estudos, foi realizada uma seleção inicial com a análise de títulos, resumos e palavras-chave dos documentos recuperados. Posteriormente, a leitura examinatória do texto completo dos documentos, e para finalizar, foi analisada a lista de referências com a finalidade de identificar estudos adicionais a partir do seu texto completo. Caso acontecesse algum desacordo entre os pares de pesquisadores, um terceiro pesquisador faria a análise do artigo recuperado e, por seu intermédio, determinaria a concordância ou discordância. Vale destacar que a leitura ocorreu em pares por pesquisadores independentes.

As evidências recuperadas foram apresentadas em um fluxograma Preferred Reporting Items for Systematic and Meta - Analyses (PRISMA - P) adaptado de Moher et al. (2009), as quais são: (1) Identificação; (2) Seleção; (3) Elegibilidade; (4) Inclusão, Figura 1.

$\mathrm{Na}$ quarta etapa, as evidências sobre as atuações do ACS foram selecionadas e agrupadas de acordo com o autor/local/ano de publicação, objetivo, método, população, tipo de produção.

E na última etapa, os revisores iniciaram a interpretação dos resultados encontrados provenientes da atuação do ACS para as ações de controle da hanseníase, descrição dos resultados esperados, alcançados e/ou não alcançados e desafios. As evidências foram interpretadas e relacionadas de acordo com cada atributo da APS, segundo referencial teórico de Starfield (2002).

\section{Resultados}

Foram identificadas 359 evidências científicas, das quais 44 (12,2\%) foram excluídas por duplicidade segundo ferramenta para gerenciamento de artigos denominada StArt. A seleção de título, resumo e palavras-chave foi completamente realizada para 315 evidências (87,7\%), sendo que 291(81\%) foram excluídos após a aplicação dos critérios de inclusão e exclusão, resultando em 24(6,6\%) que foram lidos na íntegra, resultando em 13 (3,6\%) evidências que compuseram a revisão de escopo (Figura 1). Ressalta-se que 12(3,3\%) documentos não foram recuperados mesmo utilizando-se uma conta institucional. 
A seguir, serão apresentadas no fluxograma PRISMA as etapas segundo Moher et al. (2009), as quais são: 1) Identificação, 2) Seleção, 3) Elegibilidade e 4) Inclusão, adaptado para o objetivo dessa revisão conforme Figura 1.

Figura 1. Fluxograma do processo de identificação, seleção, elegibilidade e inclusão das evidências científicas publicadas no período de 01 de janeiro de 1991 a 07 de abril de 2021(18h00min).
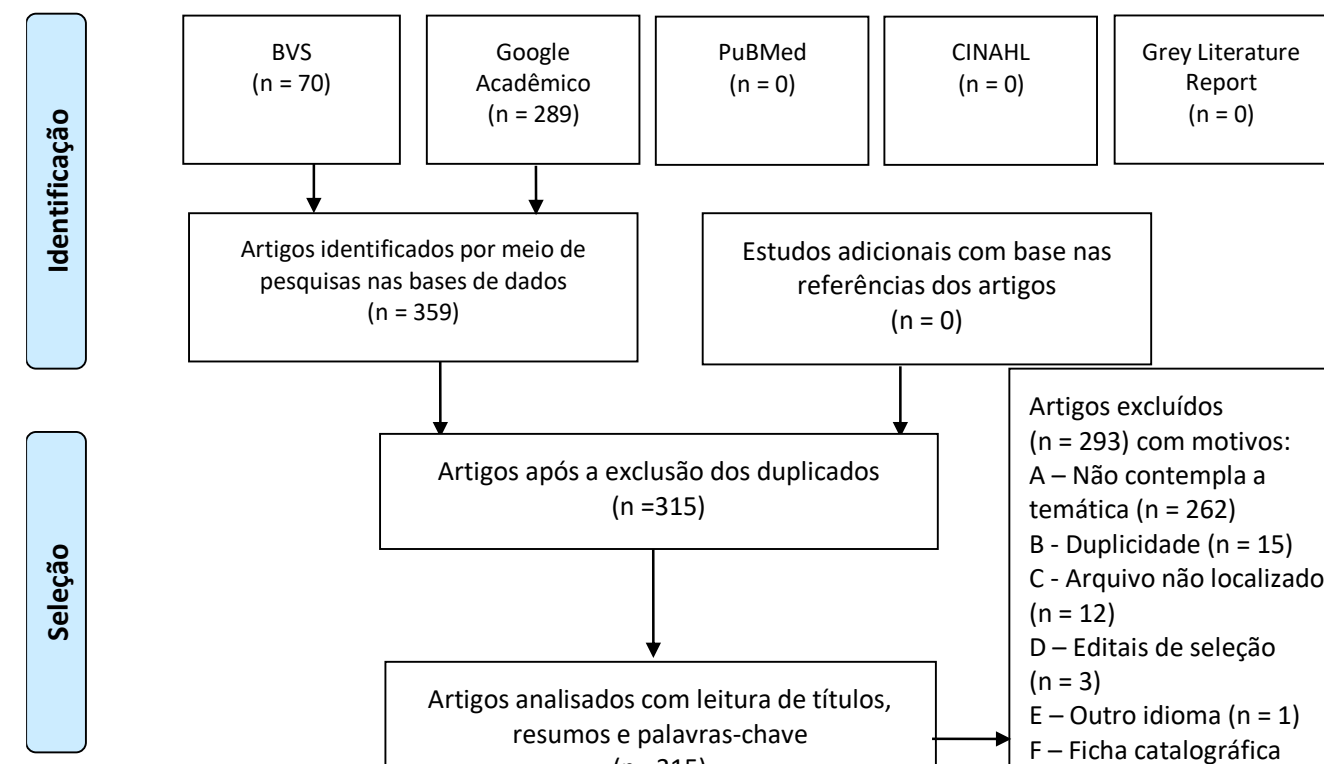

Estudos adicionais com base nas referências dos artigos

$$
(n=0)
$$
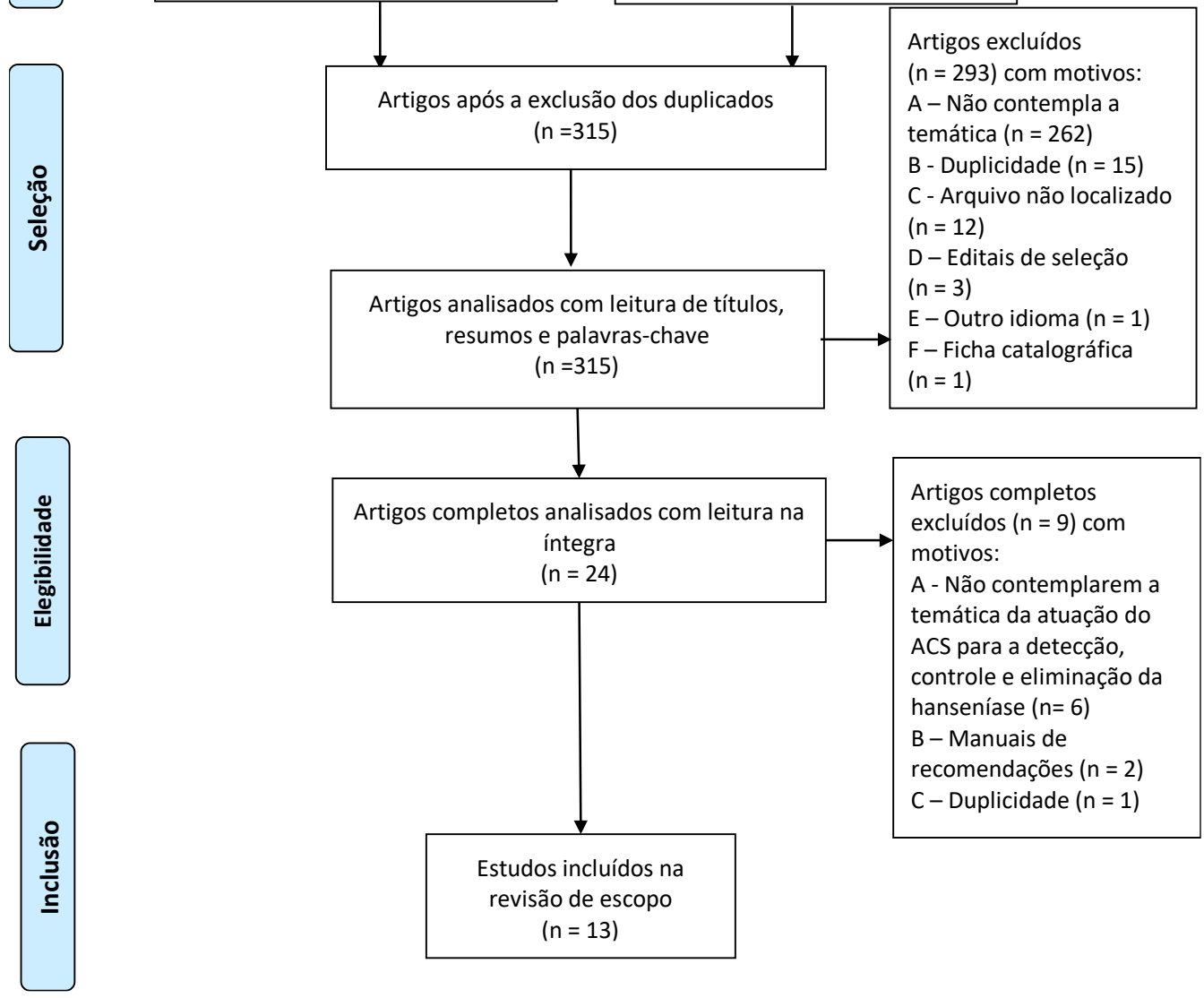

Estudos incluídos na

revisão de escopo

$(n=13)$

Fonte: Adaptado de Moher et al. (2009).

Com relação às características dos estudos incluídos e o mapeamento, pôde-se evidenciar que a maioria das pesquisas foram realizadas na Região Sudeste do Brasil, entre os anos de 2005 a 2019, correspondente ao tipo: teses, dissertações, trabalhos de conclusões de curso e artigos (Quadro 1). 
Quadro 1. Caracterização das evidências elegíveis ( $\mathrm{N}=13)$.

\begin{tabular}{|c|c|c|c|c|c|}
\hline ID & Autor/Local/ Ano & Objetivo & Método & População & Tipo de Produção \\
\hline E1 & $\begin{array}{l}\text { SANTOS, N. C Rio de } \\
\text { Janeiro - Rio de } \\
\text { Janeiro, Brasil } 2005\end{array}$ & $\begin{array}{l}\text { Analisar em que medida as práticas } \\
\text { sanitárias da ESF podem contribuir } \\
\text { para o enfrentamento da violência na } \\
\text { família. }\end{array}$ & $\begin{array}{l}\text { Estudo qualitativo, estudo } \\
\text { de caso, do tipo análise } \\
\text { situacional. }\end{array}$ & $\begin{array}{l}\text { Equipe da ESF } \\
\text { incluindo o ACS. }\end{array}$ & Tese \\
\hline E2 & $\begin{array}{l}\text { NASCIMENTO, } \\
\text { C.M.B. Recife - } \\
\text { Pernambuco, Brasil. } \\
2008\end{array}$ & $\begin{array}{l}\text { Analisar o cumprimento das } \\
\text { atividades desenvolvidas pelos agentes } \\
\text { comunitários de saúde de acordo com } \\
\text { o que é preconizado pelo Ministério da } \\
\text { Saúde. }\end{array}$ & $\begin{array}{l}\text { Estudo descritivo } \\
\text { transversal. }\end{array}$ & ACS & Dissertação \\
\hline $\mathbf{E 3}$ & $\begin{array}{l}\text { LIMA, A.B. Pedras de } \\
\text { Fogo - Paraíba, Brasil } \\
\qquad 2009\end{array}$ & $\begin{array}{l}\text { Elaborar uma análise das } \\
\text { representações sobre a hanseníase e } \\
\text { das condições sociais geradoras desta } \\
\text { doença em pacientes do município } \\
\text { paraibano de Pedras de Fogo. }\end{array}$ & Estudo qualitativo. & $\begin{array}{l}\text { Usuários do } \\
\text { centro de } \\
\text { Referência do } \\
\text { município de } \\
\text { Pedras de Fogo. }\end{array}$ & Dissertação \\
\hline E4 & $\begin{array}{l}\text { SILVA, M. M. F. São } \\
\text { Paulo - São Paulo, } \\
\text { Brasil. } 2009\end{array}$ & $\begin{array}{l}\text { Analisar a percepção dos agentes } \\
\text { comunitários de saúde sobre a } \\
\text { promoção da saúde, a partir da sua } \\
\text { formação e da sua prática, e discutir } \\
\text { sobre as possibilidades e limitações da } \\
\text { atuação desses trabalhadores. }\end{array}$ & $\begin{array}{l}\text { Estudo qualitativo, } \\
\text { utilizando o método da } \\
\text { pesquisa participante } \\
\text { (pesquisa-ação). }\end{array}$ & ACS & Dissertação \\
\hline $\mathbf{E 5}$ & $\begin{array}{c}\text { SCERNI, R. B. } \\
\text { Ceilância -Distrito } \\
\text { Federal, Brasil } 2013\end{array}$ & $\begin{array}{l}\text { Identificar a prevalência de Diabetes } \\
\text { Mellitus e de Hipertensão Arterial e o } \\
\text { perfil dos portadores destas patologias } \\
\text { na comunidade. }\end{array}$ & $\begin{array}{l}\text { Estudo observacional, } \\
\text { descritivo e de natureza } \\
\text { quantitativa. }\end{array}$ & $\begin{array}{l}\text { Informações de } \\
\text { usuários contidas } \\
\text { nas fichas } \\
\text { eletrônicas }\end{array}$ & TCC graduação \\
\hline E6 & $\begin{array}{l}\text { CANÁRIO, D. D. R. C; } \\
\text { COSTA e SILVA, S.P; } \\
\text { COSTA, F. M. Petrolina } \\
\text { - Pernambuco, Brasil. } \\
2014\end{array}$ & $\begin{array}{l}\text { Avaliar o conhecimento de Agentes } \\
\text { Comunitários de Saúde (ACS) acerca } \\
\text { da hanseníase. }\end{array}$ & $\begin{array}{l}\text { Estudo descritivo e } \\
\text { exploratório, com } \\
\text { abordagem qualitativa. }\end{array}$ & ACS & Artigo \\
\hline E7 & $\begin{array}{c}\text { LANZA, F. M. } \\
\text { Almenara, Teófilo } \\
\text { Otoni e Governador } \\
\text { Valadares - Minas } \\
\text { Gerais, Brasil } \\
2014\end{array}$ & $\begin{array}{l}\text { Avaliar os atributos da APS na } \\
\text { atenção à hanseníase nos municípios } \\
\text { de Almenara, Teófilo Otoni e } \\
\text { Governador Valadares, Minas Gerais. }\end{array}$ & $\begin{array}{l}\text { Pesquisa avaliativa; Diário } \\
\text { de campo. }\end{array}$ & $\begin{array}{c}\text { Equipes da ESF } \\
\text { incluindo o ACS e } \\
\text { usuários }\end{array}$ & Tese \\
\hline E8 & $\begin{array}{l}\text { SANTOS, J.S. et al. } \\
\text { Passos - Minas Gerais, } \\
\text { Brasil } \\
2014\end{array}$ & $\begin{array}{l}\text { Conhecer o conceito de promoção da } \\
\text { saúde dos agentes comunitários de } \\
\text { saúde de equipes de saúde da família. }\end{array}$ & $\begin{array}{c}\text { Estudo descritivo e } \\
\text { exploratório, de abordagem } \\
\text { qualitativa. }\end{array}$ & $\begin{array}{l}\text { ACS das equipes } \\
\text { de Saúde da } \\
\text { Família de Passos- } \\
\text { Minas Gerais }\end{array}$ & Artigo \\
\hline E9 & $\begin{array}{l}\text { LIMA, R. S. K. et al. } \\
\text { Limeira de Mantena - } \\
\text { Minas Gerais, Brasil. } \\
\quad 2016\end{array}$ & $\begin{array}{l}\text { Realizar a busca ativa de novos casos } \\
\text { da doença em um distrito do município } \\
\text { de Mantena, e caracterizar a situação } \\
\text { socioeconômica e epidemiológica da } \\
\text { hanseníase na região. }\end{array}$ & $\begin{array}{l}\text { Estudo descritivo, com } \\
\text { abordagem quantitativa. }\end{array}$ & $\begin{array}{l}\text { Moradores do } \\
\text { distrito de Limeira } \\
\text { de Mantena - } \\
\text { MG, Brasil. }\end{array}$ & Artigo \\
\hline E10 & $\begin{array}{l}\text { ROMANHOLO, H. S. } \\
\text { B. et al. Cacoal - } \\
\text { Rondônia, Brasil. } 2018\end{array}$ & $\begin{array}{l}\text { Caracterizar os padrões da abordagem } \\
\text { de contatos intradomiciliares de } \\
\text { hanseníase. }\end{array}$ & $\begin{array}{c}\text { Estudo transversal e } \\
\text { descritivo. }\end{array}$ & $\begin{array}{l}\text { Contatos } \\
\text { intradomiciliares } \\
\text { de hanseníase }\end{array}$ & Artigo \\
\hline E11 & $\begin{array}{l}\text { TAVARES, J. B. et al. } \\
\text { Tucuruí - Pará, Brasil. } \\
\qquad 2019\end{array}$ & $\begin{array}{l}\text { Descrever a aplicação dos passos da } \\
\text { metodologia da problematização como } \\
\text { recurso facilitador do aprendizado a } \\
\text { partir de experiências e realidades } \\
\text { vividas em dois serviços de saúde que } \\
\text { atendem usuários com hanseníase. }\end{array}$ & $\begin{array}{l}\text { Estudo descritivo, com } \\
\text { relato de } \\
\text { experiência sobre os } \\
\text { desdobramentos da } \\
\text { Metodologia da } \\
\text { Problematização com uso } \\
\text { do Arco de Maguerez. }\end{array}$ & $\begin{array}{c}\text { Profissionais } \\
\text { enfermeiros } \\
\text { responsáveis pela } \\
\text { equipe e os ACS } \\
\text { de dois centros de } \\
\text { saúde da cidade } \\
\text { de Tucuruí - Pará, } \\
\text { Brasil. }\end{array}$ & Artigo \\
\hline E12 & $\begin{array}{l}\text { HERNANDEZ, R. T. } \\
\text { Nova Olinda do Norte - } \\
\text { Amazonas, Brasil. } 2019\end{array}$ & $\begin{array}{l}\text { Estabelecer estratégias para detectar e } \\
\text { tratar precocemente os casos novos da } \\
\text { hanseníase no município de Nova } \\
\text { Olinda do Norte. }\end{array}$ & Projeto de intervenção. & $\begin{array}{l}\text { Profissionais da } \\
\text { saúde incluindo o } \\
\text { ACS }\end{array}$ & $\begin{array}{c}\text { TCC } \\
\text { Pós-graduação }\end{array}$ \\
\hline E13 & $\begin{array}{l}\text { SOUZA, F. A. M. } \\
\text { Itaboraí - Rio de } \\
\text { Janeiro, Brasil. } 2019\end{array}$ & $\begin{array}{l}\text { Investigar as percepções e práticas } \\
\text { dos profissionais de saúde que possam } \\
\text { contribuir para a superação das } \\
\text { adversidades na área psicossocial } \\
\text { enfrentadas por crianças, adolescentes } \\
\text { e suas famílias, no âmbito da atenção } \\
\text { primária proposta pela Estratégia de } \\
\text { Saúde da Família (ESF) do município } \\
\text { de Itaboraí. }\end{array}$ & $\begin{array}{l}\text { Trata-se de uma pesquisa } \\
\text { de abordagem qualitativa. }\end{array}$ & $\begin{array}{l}\text { Profissionais da } \\
\text { saúde, incluindo o } \\
\text { ACS }\end{array}$ & Dissertação \\
\hline
\end{tabular}

Nota: TCC $=$ Trabalho de Conclusão de Curso. Fonte: Autores. 
Realizou-se as descrições das atuações do ACS e os principais resultados esperados, alcançados e/ou não alcançados (Quadro 2).

Quadro 2. Descrição das atuações dos Agentes Comunitários de Saúde para as ações de controle da hanseníase e resultados evidenciados.

\begin{tabular}{|c|c|c|}
\hline \multicolumn{2}{|r|}{ ATUAÇÃO DO ACS } & \multirow{2}{*}{$\begin{array}{l}\text { DESCRIÇÃo DOS RESULTADOS ESPERADOS, ALCANÇADOS E/OU } \\
\text { NÃo ALCANÇADOS E DESAFIOS } \\
\text { Os autores não descreveram os resultados relacionados à atuação dos ACS } \\
\text { frente à busca ativa. }\end{array}$} \\
\hline E1 & 1. Busca Ativa & \\
\hline E2 & $\begin{array}{l}\text { 1. Busca ativa; } \\
\text { 2. Acompanhamento e supervisão da dose diária; } \\
\text { 3. Questionamentos de reações indesejáveis; } \\
\text { 4. Verificação de data da última consulta; } \\
\text { 5. Anotação dos resultados dos exames; } \\
\text { 6. Busca ativa de contatos intradomiciliares; } \\
\text { 7. Encaminhamento para exames. }\end{array}$ & Não se observou o engajamento de todos os profissionais, inclusive o ACS. \\
\hline $\mathbf{E 3}$ & $\begin{array}{l}\text { 1. Entrega de medicamentos em domicílio para os faltosos } \\
\text { 2. Orientações sobre a hanseníase }\end{array}$ & Evitou falhas no tratamento medicamentoso. \\
\hline E4 & $\begin{array}{l}\text { 1. Registro das ações em fichas } \\
\text { 2. Busca ativa de casos de hanseníase } \\
\text { 3. Grupo de hanseníase }\end{array}$ & $\begin{array}{l}\text { Contribuições para instrumentalizar os processos de formação e educação } \\
\text { permanente dos ACS no Brasil. }\end{array}$ \\
\hline E5 & 1. Cadastramento do hanseniano & $\begin{array}{l}\text { O estudo não apresenta resultados relacionados ao trabalho dos ACS frente à } \\
\text { hanseníase. }\end{array}$ \\
\hline E6 & 1. Participação em capacitação/Treinamento sobre a hanseníase & $\begin{array}{l}\text { Aprimoramento dos conhecimentos sobre hanseníase e importância de detectar } \\
\text { sinais e sintomas da hanseníase na comunidade e orientação sobre o tratamento } \\
\text { contínuo. }\end{array}$ \\
\hline E7 & $\begin{array}{l}\text { 1. Administração da dose diária da poliquimioterapia; } \\
\text { 2. Acompanhamento após a alta por cura; } \\
\text { 3. Treinamento sobre doenças prevalentes quando assumiram o } \\
\text { cargo. }\end{array}$ & Acompanhamento do caso de hanseníase. \\
\hline E8 & $\begin{array}{l}\text { 1.Detecção de casos de hanseníase; } \\
\text { 2.Encaminhamento ao posto de saúde }\end{array}$ & $\begin{array}{l}\text { Alguns ACS possuem uma visão da promoção à saúde relacionada a questões } \\
\text { da Carta de Ottawa (determinantes sociais, intersetorialidade e participação } \\
\text { comunitária) } \\
\text { Conceituaram promoção à saúde relacionada à prevenção de doenças crônicas, } \\
\text { incluindo a hanseníase. }\end{array}$ \\
\hline E9 & $\begin{array}{l}\text { 1. Participação em reuniões que obtiveram esclarecimentos sobre } \\
\text { lesões cutâneas que pudessem ser suspeitas de hanseníase. }\end{array}$ & $\begin{array}{l}\text { Identificação de novos casos melhora na qualidade da assistência prestada pelo } \\
\text { ACS. }\end{array}$ \\
\hline E10 & 1. Vigilância de contatos & $\begin{array}{l}\text { Falta orientações sobre precisas sobre a vacina BCG, retorno à unidade e } \\
\text { informações sobre a doença. }\end{array}$ \\
\hline E11 & $\begin{array}{l}\text { 1. Participação na pesquisa de observação dos pesquisadores } \\
\text { reflexão sobre a realidade vivenciada. } \\
\text { 2. Colaboração na definição de pontos chaves para se atingir o } \\
\text { objetivo de eliminação da hanseníase: } \\
\text { 3. Busca ativa; } \\
\text { 4. Educação em saúde. }\end{array}$ & $\begin{array}{l}\text { Contribuições do ACS na produção de uma cartilha, desenvolvida pelos } \\
\text { pesquisadores } \\
\text { pesquisadores. } \\
\text { Participação da Semana da Hanseníase para os escolares. }\end{array}$ \\
\hline E12 & $\begin{array}{l}\text { 1. Participações em palestras sobre o tema hanseníase; } \\
\text { 2. Distribuição de panfletos e orientação à comunidade; } \\
\text { 3.Realização de visitas domiciliares; } \\
\text { 4. Educação em saúde para as escolas. }\end{array}$ & $\begin{array}{l}\text { Aumento do número de casos novos notificados } \\
\text { Sensibilização da comunidade. }\end{array}$ \\
\hline E13 & 1. Educação em Saúde na escola & $\begin{array}{l}\text { Os autores não descreveram os resultados relacionados a atuação dos ACS } \\
\text { frente à educação em saúde }\end{array}$ \\
\hline
\end{tabular}

Fonte: Elaborado pelos autores.

Na sequência, com base na atuação do ACS para as ACH foram categorizadas de acordo com os atributos da APS Quadro 3. 
Quadro 3. Categorização dos atributos da APS na realização das ACH contemplados nas evidências incluídas na revisão de escopo.

\begin{tabular}{|l|l|}
\hline \multicolumn{1}{|c|}{ Atributo } & E1, E2, E8, E11 \\
\hline Porta de Entrada & Não encontrado \\
\hline Acesso & E2, E3, E6, E7 \\
\hline $\begin{array}{l}\text { Atendimento Continuado } \\
\text { Integralidade dos Serviços Disponíveis e } \\
\text { Prestados }\end{array}$ & E8, E10 \\
\hline Orientação Familiar & E3, E7 \\
\hline Orientação Comunitária & E6, E7, E9, E11, E12, E13 \\
\hline Orientação Profissional & E4, E6, E7, E9, E11, E12 \\
\hline & Fonte: Elaborado pelos autores. \\
\hline
\end{tabular}

\section{Discussão}

Essa revisão se propôs a mapear e descrever as evidências científicas sobre a atuação do ACS frente às ações de controle da hanseníase e categorizá-las segundo os atributos da APS. Embora as evidências científicas apresentem atuações que contemplem um ou mais atributos (essenciais ou derivados), para Mendes (2012) uma assistência prestada de forma efetiva, eficiente, centrada no usuário e família poderá ser atingida quando, os seus sete atributos estiverem sendo operacionalizados em sua totalidade.

Chama a atenção para o ano da publicação/divulgação dos estudos começarem a partir de 2005, devido a preocupação mundial em se combater a doença há décadas e devido à inserção do ACS iniciar em 1991. Além disso, observa-se que a maior parte dos estudos foram realizados na Região Sudeste do país, que pode estar relacionado à concentração de programas de pósgraduação nessa região, visto que boa parte das pesquisas eram produções dos programas Stricto Sensu, resultando em um desequilíbrio entre as produções nacionais conforme conhecido (Sidone et al., 2016).

A seguir, serão apresentadas as atuações dos ACS para as ACH evidenciadas nos estudos elegíveis para essa revisão de acordo com o referencial teórico de Starfield (2002).

\section{Categoria 1 - Atributos Essenciais}

\section{Porta de Entrada}

A relação dos usuários que procuram a unidade em casos de reconhecimento de um novo problema de saúde ou um novo advento de uma doença preexistente de modo espontâneo ou por encaminhamento do ACS. Esse comportamento é primordial para a vinculação da população à unidade básica de saúde e consolidação da APS (Sousa et al., 2017).

Nos estudos elegíveis, foram constatadas evidências como: cadastramento de casos de hanseníase; detecção de sinais e sintomas na comunidade; Visita domiciliar para detecção de novos casos; aplicação de um questionário com perguntas sobre sinais e sintomas da hanseníase. Nessa direção, a literatura considera importante fortalecer as ações para afecções de pele para a comunidade adscrita bem como realizar uma triagem com esse enfoque para evitar casos subdiagnosticados (Lopes, \& Lavor et al., 2020).

\section{Acesso de Primeiro Contato}

Esse atributo não foi evidenciado nas evidências recuperadas sobre a atuação do ACS.

A responsabilização pela garantia do transporte até o estabelecimento de saúde é fundamental para assegurar a universalidade, igualdade e equidade dos usuários, principalmente para os que possuem dificuldade de locomoção (Almeida et al., 2020). 
Nessa direção, ampliação do acesso alimenta a prestação de serviços de saúde e nos remete à reflexão da consolidação do SUS nas décadas de 1990 e 2000 que tem como princípio fundamental, a universalização (Rosário et al., 2020).

\section{Atendimento Continuado (Longitudinalidade)}

Considerando este atributo no acompanhamento do doente de hanseníase, o ACS realiza "o exercício de atividades de prevenção de doenças e de promoção da saúde (...) mediante ações domiciliares ou comunitárias, individuais ou coletivas, desenvolvidas em conformidade com as diretrizes do SUS" (Lei n $\left.{ }^{\circ} 13.595,2018\right)$.

Nesse espectro os estudos elegíveis (E2, E3, E6, E7) evidenciaram que o acompanhamento tem sido realizado pelos ACS, quanto à adesão ao tratamento, verificação de reações indesejadas e supervisão da dose diária. Após o diagnóstico da doença, o serviço de saúde deve assegurar a atenção integral à saúde dentro da longitudinalidade do cuidado, visando os objetivos que vão além da cura da doença (Souza et al., 2018).

Em consonância com esses dados, Brasil (2017) recomenda a continuidade da relação dos cuidados para fortalecer o vínculo entre profissionais e usuários de modo contínuo.

\section{Integralidade dos Serviços Disponíveis e Prestados}

No catálogo de serviços disponíveis e/ou prestados, o serviço de saúde deverá ofertar atenção integral partindo do pressuposto biopsicossocial como ações de promoção, prevenção, cura e reabilitação (Brasil, 2020). Dentre os serviços disponíveis pela APS e/ou prestados, destaca-se a evidência de vigilância dos contatos intradomiciliares, realizada pelos profissionais incluindo o ACS (E10).

Para contemplar a integralidade, um estudo evidenciou que os enfermeiros reforçaram a necessidade de qualificação da equipe da APS com vistas à deteç̧ão oportuna da doença e prevenção de incapacidades físicas. Além disso, reconheceu o preconceito como a maior barreira para a completude desse atributo, tornando necessária a atuação da equipe multidisciplinar completa como elemento fortalecedor no enfrentamento da hanseníase pelo doente e seus familiares (Albano et al., 2020).

\section{Categoria 2 - Atributos Derivados (Qualificadores) \\ Orientação Familiar}

No que concerne à orientação familiar para as ações de controle da hanseníase, a evidência E3 descreve a realização de orientações sobre o tratamento contínuo e visitas domiciliares para a realização de orientações sobre a doença.

Nesse sentido, um estudo realizado por Ribeiro et al. (2017) constatou que a falha no tratamento para a doença pode ser minimizada por meio de orientações e esclarecimentos sobre a poliquimioterapia e os efeitos indesejáveis realizadas por todos os membros da ESF. Além disso, ressalta a importância de considerar e incentivar o familiar como coadjuvante no tratamento.

\section{Orientação Comunitária}

Partindo das atribuições requeridas ao ACS descritas no único manual até a atualidade Brasil (2009) - "Guia prático do agente comunitário de saúde", recomenda o desenvolvimento de ações de educação em saúde e mobilização da comunidade em instituições de saúde tais como: escolas, conselhos de saúde, associação de moradores e etc... deverão ser realizadas abordando a importância do autoexame dermatológico, controle da hanseníase e combate ao preconceito.

Essa revisão evidenciou que tem sido realizadas reuniões para grupos que abordaram temas como detecção e autoexame, além disso, foram realizadas sensibilizações nas escolas bem como a produção de material didático pela equipe do ensino e serviço, incluindo o ACS (E6, E9, E11, E12, E13). 
Sob essa ótica, um estudo brasileiro realizado com adolescentes, aponta que os conhecimentos equivocados sobre a hanseníase advêm principalmente dos familiares favorecendo o estigma e o preconceito social. O fortalecimento de estratégias de educação em saúde para a comunidade poderá ultrapassar essas barreiras (Freitas et al., 2019).

\section{Orientação profissional}

Com relação a esse atributo validado por Lanza (2014), considera-se que esse aspecto seja necessário nos serviços de saúde e atendimento prestado, visto que os profissionais do SUS têm acesso a diversas capacitações e/ou qualificações.

Com base na literatura, a orientação profissional está intimamente ligada ao processo de ascensão das metas para o enfrentamento da hanseníase (Brasil, 2019).

A maioria dos estudos elegíveis (E4, E6, E7, E9, E11 e E12) para essa revisão apontaram a integração do ensinoserviço para a qualificação profissional. Foram evidenciados treinamentos abrangentes sobre a temática em questão e ainda, treinamentos sobre classes de doenças incluindo a hanseníase, observando impactos positivos na formação profissional e devolutiva para a comunidade.

A despeito disso, uma revisão realizada recentemente demonstrou como resultado desafios a serem superados sobre a formação dos ACS acerca da hanseníase, tímida ao cenário epidemiológico da doença (Araújo et al., 2017).

Com relação à integração da equipe nesse processo, um estudo realizado no nordeste brasileiro descreve a realização de oficinas destinada ao ACS para as ações de controle da hanseníase evidenciaram que esse profissional requer um suporte da equipe ESF quando se depara com situações adversas e particularidades relacionadas à doença (Lopes, \& Silva et al., 2020).

Nesse contexto, a integração da equipe pode evitar fragmentação das ações da ESF. Denota-se que há pouca visibilidade desse profissional, inclusive, no que tange à inserção e escuta pela equipe, pouca participação nas situações decisórias que reflete na redução do processo crítico e de comprometimento com as ações na comunidade (Maciazeki-Gomez et al., 2016).

O processo de sintetizar as informações dessa revisão apresentou limitações acerca da atuação do ACS pois, a maioria dos estudos apresentavam as ações de controle de diversas doenças prevalentes e não somente sobre a hanseníase.

\section{Considerações Finais}

De forma a atender o objetivo desse estudo, a revisão de escopo possibilitou a compreensão de como a atuação do ACS para as ações de controle da hanseníase vem sendo aplicada nos diferentes cenários, transparecendo um olhar diferenciado sobre as estratégias de controle desse agravo.

Os resultados evidenciaram a real necessidade de capacitação desse profissional, nos convocando a refletir sobre as ferramentas subsidiadas para realizarem a atuação com qualidade. Por outro lado, foi destacada a importância da sua atuação junto ao indivíduo, família, grupos e sociedade na magnitude dos usuários e pesquisadores.

Por fim, sugere-se que estudos de intervenções sejam realizados em diferentes cenários nacionais e internacionais para aumentar a divulgação científica: acadêmica e para além da comunidade acadêmica, capacitando e ressaltando a importância desse profissional no contexto da hanseníase, quanto às medidas de prevenção, controle e monitoramento dos casos e ainda, sua contribuição para a diminuição do estigma frente à doença.

\section{Referências}

Albano, M. L., Sousa, A. A. S. Roscoche, K. G. C., Oliveira Filho, P. R. V. de., \& Felício, J. F. (2020). Barreiras à integralidade do cuidado à pessoa com hanseníase: percepção de enfermeiros. Research, Society and Development, 9(8), e531985864-e531985864. http://dx.doi.org/10.33448/rsd-v9i8.5864. 
Almeida, A. P. S. C., Nunes, B. P, Duro, S. M. S., Lima, R. C. D., \& Facchini, L. A. (2020). Falta de acesso e trajetória de utilização de serviços de saúde por idosos brasileiros. Ciência \& Saúde Coletiva [online], 25(6), 2213-2226. https://doi.org/10.1590/1413-81232020256.27792018.

Araújo, E. T. H., Almeida, C. A. P. L., Sá, G. G. M., \& Moura, L. K. B. (2017). Produção científica da formação e atuação do agente comunitário de saúde sobre hanseníase e tuberculose. Revista Prevenção de Infecção e Saúde, 3(4), 46-56.

Arksey, H., \& O'malley, L. (2005). Estudos de escopo: rumo a uma estrutura metodológica. International Journal of Social Research Methodoly, 8(1), 19-32.

Brasil. (2021). Boletim epidemiológico: hanseníase, 2021. Ministério da Saúde. http://www.aids.gov.br/pt-br/pub/2021/boletim-epidemiologico-hanseniase2021.

Brasil. (2018). e-SUS Atenção Básica: manual do sistema com Coleta de Dados Simplificada: CDS - versão 3.0 [recurso eletrônico]. Ministério da Saúde. http://189.28.128.100/dab/docs/portaldab/documentos/esus/Manual_CDS_3_0.pdf.

Brasil. (2019). Estratégia nacional para o enfrentamento da hanseníase - 2019-2022. Ministério da Saúde. http://portalarquivos2.saude.gov.br/images/pdf/2019/marco/27/estrategia-nacional-cghde-consulta-publica-27mar.pdf.

Brasil. (2009). Guia prático do agente comunitário de saúde (Série A. Normas e Manuais Técnicos). Ministério da Saúde.

Brasil. (2017). Guia prático sobre a hanseníase. Ministério da Saúde.

Brasil. (2020). Manual do instrumento de avaliação da atenção primária à saúde: PCATool-Brasil - 2020. Ministério da Saúde.

Briggs, J. (2015). JBI reviewers manual: methodology for JBI-Scoping Reviews. Joanna Briggs Institute. http://joannabriggs.org/assets/docs/sumari/Reviewers-Manual_Methodology-for-JBI-Scoping-Reviews_2015_v2.pdf.

Canário, D. D. R. do C., Silva, S. P. C. e, \& Costa, F. M. da. (2014). Saberes e práticas de agentes comunitários de saúde acerca da hanseníase. Revista de Enfermagem UFPE on line, 8(1), 1-7.

Fabbri, S., Silva, C., Hernandes, E., Octaviano, F., Thommazo, A. di, \& Belgamo, A. (2016). Improvements in the StArt tool to better support the systematic review process [Conference proceedings]. 20th International Conference on Evaluation and Assessment in Software Engineering, Limerick, Ireland.

Freitas, B. H. B. M. de, Silva, F. B. e, Silva, K. F. da, Santos, H. C. D. dos, \& Silva, S. E. G. (2019). Percepção de adolescentes sobre a hanseníase. Revista de Enfermagem UFPE on line, 13(2), 292-297.

Hernandez, R. T. (2017). Estratégias para diagnóstico e tratamento precoce da hanseníase no município de nova olinda do norte-amazonas [Trabalho de Conclusão de Curso, Universidade Federal de Ciências da Saúde de Porto Alegre].

Lanza, F. M. (2014). Avaliação da atenção primária no controle da hanseníase: validação de instrumentos e análise do desempenho de municípios endêmicos do estado de Minas Gerais [Tese de doutorado, Escola de Enfermagem da Universidade Federal de Minas Gerais].

Lei $n^{\circ}$ 13.595, de 05 de janeiro de 2018. (2018). Altera a Lei ${ }^{\circ} 11.350$, de 5 de outubro de 2006, para dispor sobre uma reformulação de atribuições, uma jornada e como condições de trabalho, ou o nível de treinamento profissional, os cursos de formação técnica e continuação e indenização do transporte de profissionais Agentes Comunitários de Saúde e Agentes de Combate às Endemias. http://www.planalto.gov.br/ccivil_03/_ato2015-2018/2018/lei/L13595.htm.

Levac, D., Colquhoun, H., \& O’ Brien, K. K. (2010). Scoping studies: advancing the methodology. Implementation Science, 5(69).

Lima, A. B. (2009). O estigma do preconceito: um estudo sobre representações de pacientes portadores de hanseníase no Município de Pedras de FogoParaíba [Dissertação de mestrado, Universidade Lusófona de Humanidades e Tecnologias].

Lima, R. S. K. E., Oliveira, L. B. P. D., Gama, R. S., Ferreira, J. A. G., Grossi, M. A. de F., Fairley, J. K., Silva, F. G. da, \& Fraga, L. A. de O. (2016). A importância da busca ativa como estratégia de controle da hanseníase em territórios endêmicos. Hansenologia Internationalis, 41(1/2), 55-63.

Lopes, C. N., Lavor, M. de L. S. S., Carvalho, A. L. D. de, Damaceno, M. M. de P., Andrade, F. F. de, Leite Filho, G. de A. A., Benevolo, A. T. de, \& Lopes, O. S. (2020). A insustentável leveza do toque: aprendizagens em hanseníase. Brazilian Journal of Health Review, 3(4), 7710-7721.

Lopes, E. F. B., Silva, L. S. A. da, Rotta, C. de S., Oliveira, J. H. M. de, Menezes, I. R. de, Nakamura, L., Aoyagi, G. A., Pereira, C. S., Oliveira, R. A. M. de, Menezes, L. L. B., Moreira, L. B., Assis, M. de F. B. R. de, \& Simões, E. A. P. (2020). Educação em saúde: uma troca de saberes no combate ao estigma da hanseníase/Health education: exchange of knowledge in combat the stigma of leprosy. Brazilian Journal of Development, 6(2), 5350-5368.

Maciazeki-Gomes, R. D. C., Souza, C. D. de, Baggio, L., \& Wachs, F. (2016, Maio). O trabalho do agente comunitário de saúde na perspectiva da educação popular em saúde: possibilidades e desafios. Ciência \& Saúde Coletiva, 21(5), 1637-1646.

Mendes, E. V. (2012). O cuidado das condições crônicas na atenção primária à saúde: o imperativo da consolidação da estratégia da saúde da família. Organização Pan-Americana da Saúde.

Moher, D., Liberati, A., Tetzlaff, J., Altman, D. G., \& The PRISMA Group (2009). Preferred reporting items for systematic reviews and meta-analyses: the PRISMA statement. PLoS Med, 6(7):e1000097. 10.1371/journal.pmed.1000097.

Nascimento, C. M. B. D. (2008). Análise do cumprimento das práticas dos agentes comunitários de saúde em municípios da Região Metropolitana do Recife [Dissertação de mestrado, Centro de Pesquisas Aggeu Magalhães, Fundação Oswaldo Cruz].

Peruzzo, H. E., Bega, A. G., Lopes, A. P. A. T., Haddad, M. do C. F. L., Peres, A. M., \& Marcon, S. S. (2018). Os desafios de se trabalhar em equipe na estratégia saúde da família. Escola Anna Nery, 22(4), e20170372.

Portaria $n^{\circ}$ 2436, de setembro de 2017. (2017). Aprova a Política Nacional de Atenção Básica, estabelecendo a revisão de diretrizes para a organização da Atenção Básica, no âmbito do Sistema Único de Saúde (SUS). https://bvsms.saude.gov.br/bvs/saudelegis/gm/2017/prt2436_22_09_2017.html. 
Resolução $n^{\circ}$ 573, de 31 de janeiro de 2018. (2018). Resolve aprovar o Parecer Técnico no 28/2018 contendo recomendações do Conselho Nacional de Saúde (CNS) à proposta de Diretrizes Curriculares Nacionais (DCN) para o curso de graduação Bacharelado em Enfermagem. https://www.in.gov.br/materia//asset_publisher/Kujrw0TZC2Mb/content/id/48743098/do1-2018-11-06-resolucao-n-573-de-31-de-janeiro-de-2018-48742847.

Ribeiro, M. D. A., Castillo, I. da S., Silva, J. C. A., \& Oliveira, S. B. (2017). A visão do profissional enfermeiro sobre o tratamento da hanseníase na atenção básica. Revista Brasileira em Promoção da Saúde, 30(2), 221-228.

Romanholo, H. S. B., Souza, E. A. de, Ramos Júnior, A. N., Kaiser, A. C. G. C. B., Silva, I. O. da, Brito, A. L., \& Vasconcellos, C. (2018). Vigilância de contatos intradomiciliares de hanseníase: perspectiva do usuário em município hiperendêmico. Revista Brasileira de Enfermagem, 71(1), 75-181.

Rosário, C. A., Baptista, T. W. de F., \& Matta, G. C. (2020). Sentidos da universalidade na VIII Conferência Nacional de Saúde: entre o conceito ampliado de saúde e a ampliação do acesso a serviços de saúde. Saúde em Debate [online], 44(124), 17-31. https://doi.org/10.1590/0103-1104202012401.

Santos, J. S., Maia, M. A. C., Andrade, R. D., \& Oliveira, C. T. (2014). Promoção da saúde na estratégia saúde da família: visão dos agentes comunitários de saúde. Revista de Enfermagem UFPE on line, 8(7, supl. 1), 2316-2323.

Santos, N. C. D. (2005). Práticas sanitárias frente à violência intrafamiliar no âmbito do PSF: um estudo de caso [Tese de doutorado, Escola Nacional de Saúde Pública da Fundação Oswaldo Cruz].

Scerni, R. B. (2013). Perfil dos usuários com hipertensão e diabetes acompanhados pelos agentes comunitários de saúde em uma área de Ceilândia-DF [Trabalho de Conclusão de Curso, Faculdade de Ceilândia, Universidade de Brasília].

Sidone, O. J. G., Haddad, E. A., \& Mena-Chalco, J. P. (2016). A ciência nas regiões brasileiras: evolução da produção e das redes de colaboração científica. Transinformação, 28(1), 15-32. http://www.scielo.br/scielo.php?script=sci_arttext\&pid=S0103-37862016000100015\&lng=pt\&nrm=iso.

Silva, M. M. F. D. (2009). Promoção da saúde: percepção dos agentes comunitários de saúde a partir da sua formação e da sua prática [Dissertação de mestrado, Universidade de São Paulo].

Sousa, G. S. de, Silva, R. L. F. da, \& Xavier, M. B. (2017). Atributos da atenção primária em saúde no controle da hanseníase: ótica do enfermeiro. Revista Baiana de Enfermagem, 31(1), e17251.

Souza, E. A., Boigny, R. N., Ferreira, A. F., Alencar, C. H., Oliveira, M. L. W., \& Ramos Júnior, A. N. (2018). Vulnerabilidade programática no controle da hanseníase: padrões na perspectiva de gênero no Estado da Bahia, Brasil. Cadernos de Saúde Pública, 34(1), p. e00196216.

Souza, F. A. M. D. (2019). Resiliência e promoção da saúde: as percepções e práticas de profissionais de saúde da estratégia saúde da família na atuação com crianças, adolescentes e suas famílias [Dissertação de mestrado, Universidade Federal Fluminense].

Starfield, B. (2002). Atenção primária: equilíbrio entre necessidades de saúde, serviços e tecnologia. UNESCO; Ministério da Saúde.

Tavares, J. B., Costa, J. L. D., Costa, J. B. da, \& Furtado, L. G. S. (2019). Método problematizador no trabalho de busca ativa de hanseníase em dois centros de saúde: descrição de experiência. Revista Brasileira de Educação e Saúde, 9(2), 1-6.

Tricco, A. C., Lillie, E., Zarin, W., O'Brien, K. K., Colquhoun, H., Levac, D., Moher, D., Peters, M. D. J., Horsley, T., Weeks, L., Hempel, S., Akl, E. A., Chang, C., McGowan J., Stewart, L., Hartling, L., Aldcroft, A., Wilson, M. G., Garritty, C., \& Straus, S. E. (2018). PRISMA extension for scoping reviews (PRISMA-ScR): checklist and explanation. Annals of Internal Medicine, 169(7), 467-473. 